\title{
Periodic structures with higher symmetries and their applications in electromagnetic devices
}

\author{
O. Quevedo-Teruel, Senior Member, IEEE, G. Valerio, Senior Member, IEEE, Z. Sipus, Senior Member, IEEE, \\ and E. Rajo-Iglesias, Senior Member, IEEE,
}

$\mathbf{H}$

IGHER SYMMETRIES frequently amaze human beings because of the illusions and incredible landscapes that they can produce. For example, imagine the unearthly pictures of the Dutch graphic artist Maurits Cornelis (M. C.) Escher. He made use of glide symmetry and reflection to produce unbelievable transitions and transformations of objects and beings, as illustrated in Fig. 1 (a). However, the history of higher symmetries started much earlier in time. M. C. Escher was partially inspired by the Moorish tessellations in the Alhambra in Granada, Spain, like the ones illustrated in Fig. 1 (b). Higher symmetries can be employed not only to create artistic creations, but also as tools for a better description of space (e.g., in connection with numerical problems in space tessellation and meshing [1] or for enhancing the performance of electromagnetic devices). Here, we explain the importance of the recently discovered electromagnetic properties of higher symmetries, as well as their implications and opportunities for microwave and antenna engineers.

\section{Higher SYMMETRIES IN THE ELECTROMAGNETIC HISTORY: BACK TO THE 60S}

Narrowing our scope to physics, higher symmetries were very popular in the 60s. For example, George L. Trigg wrote in 1965 that "in the last few months, scarcely an issue of Physical Review Letters has failed to contain at least one paper on the topic" [2], demonstrating the volume of studies at the time. If we are even more specific, and we focus our attention on electromagnetic engineering, the first studies of higher symmetries, mostly on periodically loaded waveguide structures, arrived in the middle of the 60s [3], [4], and they were popular for one decade, until the middle of the 70s [5], [6]. However, in the 70s, the electromagnetic engineering community was not ready to further develop scientific studies on higher symmetries and to understand their full potential. First, computers were very basic and there was no commercial software that could be used to simulate these complex structures. Second, our understanding of periodic structures achieved maturity in the 2000s with the arrival of the concepts of metamaterials [7], [8] and metasurfaces [9], [10]. Finally, at the end of the 70s and beginning of the 80 s, electromagnetic engineers focused their attention on making wireless systems affordable for everybody, so printed and planar technology were the selected solutions for low-cost communications at low frequencies [11].

It is in the second decade of the $21^{\text {st }}$ century, when powerful computers are easily accessible, commercial software for simulation is commonly available, and periodic structures are broadly understood thanks to studies on metamaterials and metasurfaces, when the opportunity for higher symmetries has arrived. This opportunity first arrived when the industry demanded

This work has been partly funded by the Spanish Government through project TEC2016-79700-C2-2-R, the French governement under the ANR grant HOLeYMETA ANR JCJC 2016 ANR-16-CE24-0030, the Vinnova project High-5 (2018-01522) under the Strategic Programme on Smart Electronic Systems, and by the Stiftelsen Åforsk project H-Materials (18-302).

O. Quevedo-Teruel is with the Division of Electromagnetic Engineering at KTH Royal Institute of Technology, Stockholm, Sweden (e-mail: oscarqt@kth.se).

G. Valerio is with UR2, Laboratoire d'Électronique and Électromagnétisme, Sorbonne Université, F-75005 Paris, France (e-mail: guido.valerio@ sorbonneuniversite.fr).

Z. Sipus is with the Faculty of Electrical Engineering and Computing, University of Zagreb, HR-10000 Zagreb, Croatia (e-mail: zvonimir.sipus@fer.hr).

E. Rajo-Iglesias is with the Department of Signal Theory and Communications, Carlos III University of Madrid, Spain (e-mail: eva@tsc.u3m.es).

Manuscript received January 16, 2020; revised Month XX, 2019. 


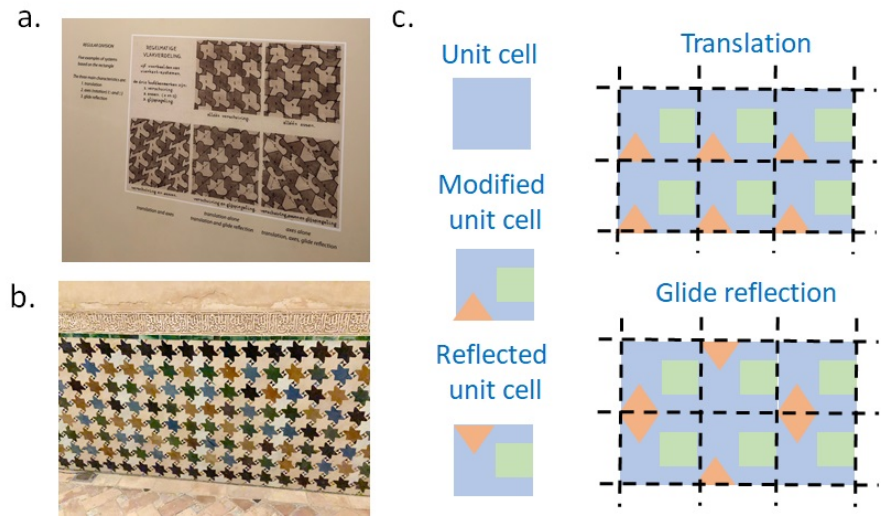

Fig. 1. Glide symmetries in art: a) Explanation of glide reflection at the museum of M. C. Escher in The Hague, The Netherlands. b) Moorish tessellations in the Alhambra, Granada, Spain. c) Compositions made of translations and glide reflection.

electromagnetic systems operating at higher frequencies [12]. At these frequency ranges, there is a need for low-loss structures that can only be achieved with fully metallic devices and integrated systems such as antennas and circuits [13].

In this situation, antennas based on leaky waves and lenses are gaining converts for communication designs in the new bands of $5 \mathrm{G}$ and satellite communications [14]. Although the design of these antennas is more complicated than arrays, they present a simpler feeding network. On the other hand, filters were traditionally designed as an independent unit that was connected to the rest of the system. Due to insertion loss, and to losses in the interconnections [15], for high-frequency designs, they must be integrated with other components. Finally, due to these large losses, future components, such as filters, must be reduced in length. Therefore, design techniques which are not based on isolated elements, but on coupled elements, are required to reduce overall losses.

\section{DEFINITION OF PERIODIC STRUCTURES WITH HIGHER SYMMETRIES}

A periodic unit cell possesses higher symmetries when it is invariant after a translation and a second geometrical variation. For example, a glide-symmetric unit cell is invariant after a translation and a mirroring [16]. A twist-symmetric unit cell is invariant after a translation and a rotation (or angular movement) [17].

\section{A. Glide symmetries}

In Fig. 1 (c), we present the creation of a periodic structure through a glide reflection. A unit cell, represented in blue, is filled with a triangle and a rectangle. One could repeat this unit cell with a simple translation, but also, create a reflected unit cell that is alternated together with the translation.

Glide symmetries can be one-dimensional or two-dimensional. In Fig. 2 (a,b), we represent two examples of one-dimensional glide-symmetric structures: corrugations [18], [19], [20] and transversal slots [21], [22]. These two structures have glide symmetry with respect to the $x$ direction; however, their mirroring planes are different. In the corrugations, this plane is horizontal, but in the slots, it is vertical. In these cases, the glide operation is $x \rightarrow x+p / 2$, and either $z \rightarrow-z$ in the corrugations, or $y \rightarrow-y$ in the slot, with $p$ as the periodicity in both cases. 


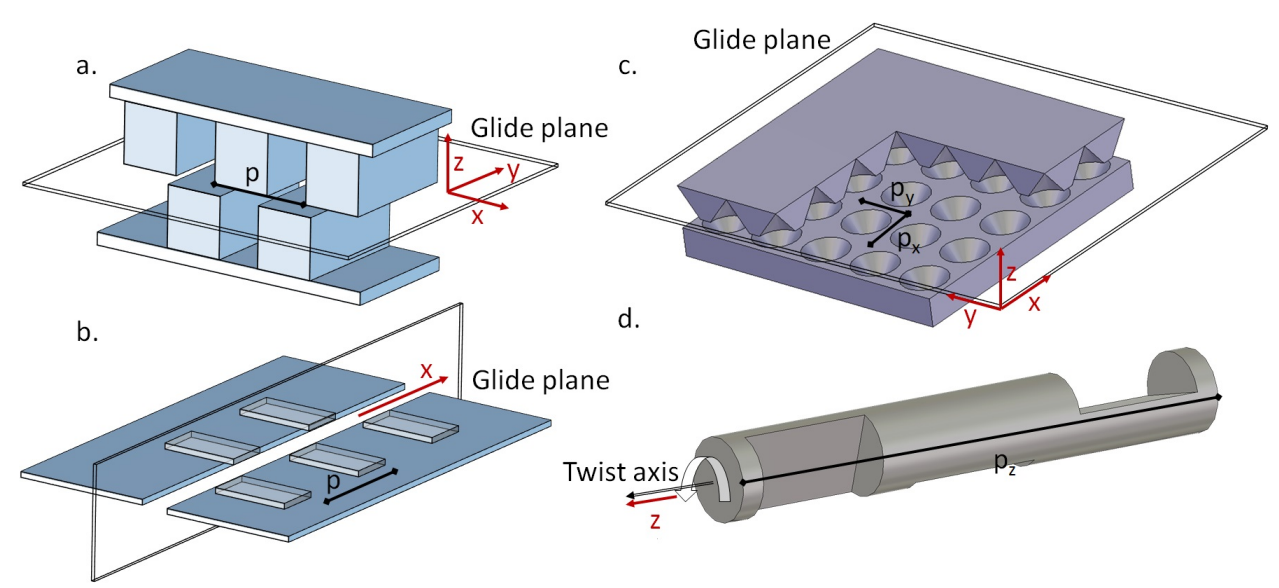

Fig. 2. Electromagnetic configurations of higher symmetries: a) Glide-symmetric corrugations. b) Slot with glide-symmetric transversal loads. c) Twodimensional glide-symmetric holey structure. d) Twist-symmetric holey configuration.

A new particular case of glide symmetry was recently reported as polar glide symmetry [17], [23]. It refers to the case in which the mirroring plane is not defined with Cartesian coordinates, but with polar ones. Therefore, the mirroring plane is orthogonal to a vector in the $\rho$ direction [23], [24].

Two-dimensional glide-symmetric structures were first studied in [25], and they require translation in two orthogonal directions that are opposite the vector of the mirroring plane. For example, a common glide operation can be $(x, y) \rightarrow$ $\left(x+p_{x} / 2, y+p_{y} / 2\right)$ and $z \rightarrow-z$ [26], [27], with $p_{x}$ and $p_{y}$ as the periods in $x$ and $y$, respectively. One example of two-dimensional glide symmetry is illustrated in Fig. 2 (c) for conical holes inserted in two parallel plates.

\section{B. Twist symmetries}

Twist-symmetric structures are those that are invariant under a translation in one direction. For example, in Fig. 2 (d), a holey metallic wire oriented in the $z$ direction is illustrated. This direction is the propagation direction. The wire has holes that rotate through the $z$ direction. A periodic structure possesses $m$-fold twist symmetry, $m$ being an integer, if it is invariant under a $p / m$ translation along and a $2 \pi / m$ rotation around the twist direction, where $p$ is the periodicity of the structure [23]. The particular case shown in Fig. 2 (d) is 3-fold. We must note that helices are a particular case of twist symmetry with $m=$ $\infty$. Helices were studied in terms of dispersion properties in the 50s [28]. For this reason, twist symmetry is also known as helical symmetry or screw symmetry [6].

\section{SUB-PERIODICITIES IN HIGHER SYMMETRIES}

Introducing higher symmetries in periodic structures may drastically change the dispersion properties of those structures (i.e., how passbands and stopbands are distributed in the frequency domain). The basic property of higher-symmetric periodic structures is the possibility to close selected bandgaps. This was already discussed by Hessel et al. [6] in the early 70s, with reference to 1-D structures. Specifically, the presence of a glide symmetry with period $p$ closes the first stopband at the edge of a Brillouin zone ( $\beta=\pi / p, \beta$ being the phase constant of a Bloch mode), and an $m$-fold twist symmetry closes the first $m-1$ stopbands, alternatively at $\beta=\pi / p$ and $\beta=0$. The same spectral properties have been more recently observed in 2-D structures [26], [27]. 
a.

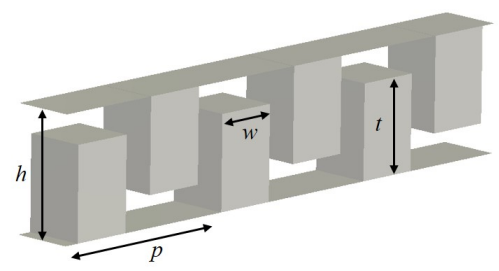

.

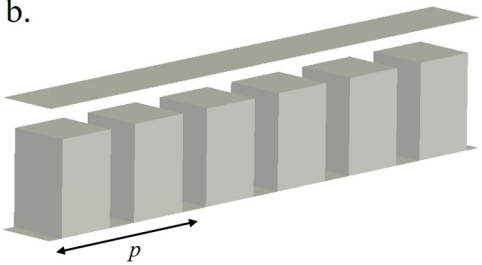

c.

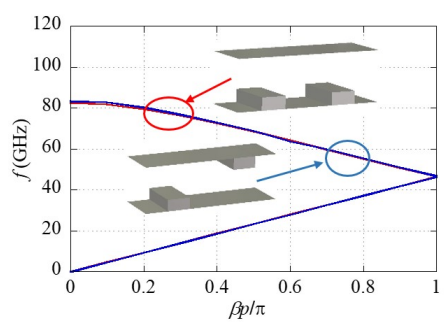

d.

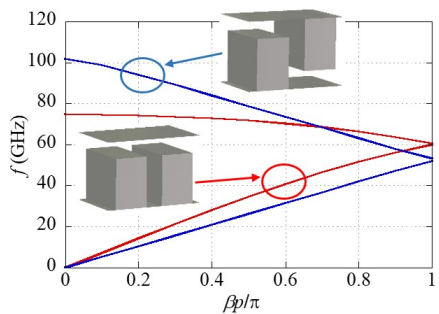

Fig. 3. a) Structure with glide-symmetric corrugations along one direction. b) Associated non-glide-symmetric structure with corrugations along one direction. (c) Brillouin diagram of glide and non-glide structures with $h=1 \mathrm{~mm}, w=0.5 \mathrm{~mm}, p=3 \mathrm{~mm}$, and $t=0.25 \mathrm{~mm}$. d) Brillouin diagram of glide and non-glide structures with $h=1 \mathrm{~mm}, w=0.5 \mathrm{~mm}, p=1.5 \mathrm{~mm}$, and $t=0.75 \mathrm{~mm}$.

For example, in a glide-symmetric structure, the absence of a stopband at $\beta=\pi / p$ can be easily explained if the effect of the periodic scatterers on the two sides of the glide plane is the same. This means that these scatterers can be moved on the same side of the plane, thus transforming the glide structure into an equivalent purely periodic structure with a halved spatial period $p / 2$. An example of such as one-dimensional glide structure and its associated non-glide are depicted in Figs. 3 (a) and (b). The Brillouin diagram of this new non-glide periodic structure presents a first stopband edge at $\beta=\pi /(p / 2)=2 \pi / p$, which is equivalent to the $\beta=0$ point in the diagram of the glide structure. In other words, the glide structure is equivalent to a structure with a shorter period, so that its first stopband is found at higher frequencies.

However, the equivalence between scatterers on the two sides of the glide plane is not necessarily verified in all glide structures. In order to explain this phenomenon, let us decompose the Bloch mode supported by the glide structure into the modes of the uniform background structure. These modes are coupled together by the periodic scatterers. In the glide structure in Fig. 3 (a), these scatterers are placed alternately on the two sides of the glide plane, while in the periodic structure of Fig. 3 (b) they are all on the same side.

On the one hand, if in both structures the scatterers are weakly interacting among one another, only one dominant mode is relevant in the coupling between adjacent scatterers. This happens if each scatterer mainly excites only one dominant background mode, or if all the background modes excited by one scatterer are strongly attenuated when they reach the adjacent ones. In this case, the mutual position of two adjacent scatterers does not impact the coupling, which is the same whether both scatterers lie on the same side of the glide plane or on opposite sides of it. The glide structure is then reducible to the non-glide periodic structure with halved spatial period [29]. An example of a reducible glide structure can be seen in Fig. 3 (c), where the glide line and the non-glide line have the same dispersion diagram, if their phase constants are normalized with respect to the same distance $p$ (the period of the glide line). On the other hand, if in at least one of the two structures in Figs. 3 (a) and (b) the scatterers are strongly interacting, several background modes will be relevant for their coupling [30]. These modes will have, in general, different parity with respect to the glide plane; the odd and the even modes will experience a different scattering 
according to the position of the scatterer with respect to the glide plane. This richer modal coupling in strongly coupled structures makes the glide-symmetric structure irreducible to the non-glide periodic one with reduced spatial period [29]. An example of an irreducible glide structure can be seen in Fig. 3 (d), where the glide and non-glide periodic structures have different dispersion diagrams. The same phenomena can be found in twisted structures, where the scatterers lie along a spiral, rather than on different sides of a plane [31].

\section{Circuit MOdels to ANALYZE GLide-SyMmetric STRUCTURES}

Despite the recent interest in higher-symmetric structures, simplified models for the efficient design of these structures are not yet available. Specific models of higher-symmetric structures are needed because the application of full-wave numerical methods to this class of structures is difficult. Interesting dispersive behaviors, such as wideband response and strong bandgap rejection, require extreme values of geometrical parameters (e.g., very close glide surfaces in order to enhance their mutual interaction). In these cases, numerical methods encounter difficulties: for example, thin gaps between surfaces require a very dense localized meshing in finite-element and finite-difference methods, or cause slow convergence in Green's functions in the method of moments. Furthermore, the study of finite structures made by a non-uniform array of thousands of sub-wavelength cells is a multiscale problem that requires ad-hoc computation techniques.

\section{A. Circuit models}

The availability of equivalent circuits for the unit cells of higher-symmetric structures would considerably simplify their preliminary design. Based on the previous analysis, if a higher-symmetric structure is reducible to a non-higher-symmetric periodic structure, a mono-modal equivalent circuit is sufficiently accurate to calculate its dispersion behavior. Furthermore, the model can be performed on only one sub-unit cell, and its non-higher-symmetric structure can be analyzed instead of the higher-symmetric structure. This was done in [19], where a one-dimensional glide-symmetric corrugated structure (as illustrated in Fig. 2(a)) was analyzed by means of an equivalent circuit derived from the T-junction discontinuity [32]. In fact, the central horizontal waveguide region can be regarded as a parallel-plate waveguide (PPW) connected with a series of vertical corrugations, each one equivalent to a PPW along the vertical direction. A unit cell can then be described as a composition of T-junctions between parallel plates. This kind of discontinuity has been widely studied and can be characterized with the closed-form scattering parameters found in [32]. The results of this equivalent circuit are extremely accurate for the full range of parameters used in current applications, as long as the unit cell keeps a T-junction topology. This is no more valid than in the glide-corrugated structure of Fig. 3(a). More recently, a circuit model analysis was proposed to model glide-symmetric loaded microstrip lines [33]. This model accurately explains the coupling effects between unit cells, and the different interactions between conventional and glide-symmetric unit cells.

\section{B. Multi-mode analysis}

When irreducible structures are of interest, a multi-modal equivalent circuit can always be used to correctly model the interactions among the different scatterers [29], [31]. In a one-dimensional higher-symmetric line, the unit cell can then be 
modeled as a $2 N$-port network, where $N$ is the number of background modes retained on each Floquet boundary of the cell in order to accurately compute the interactions between scatterers. This network can be characterized by means of its transmission matrix $\boldsymbol{T}$, whose eigenvalues are related to the wavenumbers of the Bloch modes supported by the structure. If only two modes are relevant, we get:

$$
\boldsymbol{T} \cdot\left[\begin{array}{l}
V^{(1)} \\
V^{(2)} \\
I^{(1)} \\
I^{(2)}
\end{array}\right]=e^{-j k_{x} p}\left[\begin{array}{l}
V^{(1)} \\
V^{(2)} \\
I^{(1)} \\
I^{(2)}
\end{array}\right]
$$

where $V^{(1)}$ and $I^{(1)}$ are the voltage and currents associated with the first mode on one Floquet boundary of the cell, $V^{(2)}$ and $I^{(2)}$ are the voltage and currents associated to the second mode on the same Floquet boundary, and $k_{x}$ is the (possibly complex) unknown Bloch wavenumber.

This description in terms of the transmission matrix can also lead to an alternative approach to performing a dispersion equation, by means of the multi-modal transmission matrix of a sub-cell. In this case, we need to take into account that after translating one sub-cell, each background mode composing the Bloch mode is not equivalent to a phase shift (as a translation of one cell is). In order to get a phase shift, we also need to perform a reflection [29] or a rotation [31], according to the glide or twisted nature of the structure. We can compensate each background mode for this geometrical operation by multiplying each mode by a factor depending on the mode parity. In the case of a glide line, if the first mode in (1) is even with respect to the glide plane and the second mode is odd, we get:

$$
\boldsymbol{T}_{1 / 2} \cdot\left[\begin{array}{c}
V^{(1)} \\
V^{(2)} \\
I^{(1)} \\
I^{(2)}
\end{array}\right]=e^{-j k_{x} p / 2}\left[\begin{array}{c}
V^{(1)} \\
-V^{(2)} \\
I^{(1)} \\
-I^{(2)}
\end{array}\right]
$$

where the transmission matrix $\boldsymbol{T}_{1 / 2}$ refers to only one half of the unit cell (thus allowing a faster computation). Eq. (2) confirms also that the presence of only one mode (or the presence of modes with the same parity) in both (1) and (2) makes the structure equivalent to a non-glide periodic line, having as a unit cell the sub-cell of the glide line. All these results can be easily generalized to two-dimensional glide structures [29], where background modes can be defined on each of the four Floquet boundaries of the unit cell.

\section{MODE-MATCHING TO ANALYZE GLIDE-SYMMETRIC STRUCTURES}

An appropriate way of analyzing guiding electromagnetic structures having higher symmetries is to apply the mode-matching analysis approach. Mode-matching is based on representing the electric and magnetic fields in each section of the structure as a sum of suitable modes with unknown complex amplitudes. In other words, it uses pre-knowledge about the electromagnetic 
field configuration and symmetry properties to reduce the number of unknowns. With this technique, it is possible to describe precisely the electromagnetic fields in the structure and to gain physical insight into the properties of higher symmetries.

As an example, let us consider a PPW with glide-symmetric holey walls (Figs. 2.a and 2.c). The electromagnetic fields in the PPW region (parallel to the walls) can be expressed as a series of Floquet harmonics by virtue of periodicity:

$$
\begin{aligned}
& \mathbf{E}_{t}^{\text {Gap }}=\frac{1}{p^{2}} \sum_{s, q} e^{-j\left(k_{x, s} x+k_{y, q} y\right)} \tilde{\mathbf{e}}_{t, s q}^{G a p}(z) \\
& \mathbf{H}_{t}^{\text {Gap }}=\frac{1}{p^{2}} \sum_{s, q} e^{-j\left(k_{x, s} x+k_{y, q} y\right)} \tilde{\mathbf{h}}_{t, s q}^{G a p}(z)
\end{aligned}
$$

with $k_{x, s}=k_{x, 0}+2 \pi s / p$ and $k_{y, q}=k_{y, 0}+2 \pi q / p$ by assuming a rectangular lattice with period $p$ in both the $x$ and $y$ directions. The amplitude of each Floquet harmonic of the transverse electric field can be written as

$$
\begin{aligned}
& \tilde{\mathbf{e}}_{t, s q}^{G a p}(z)=\left(\begin{array}{c}
A_{s q}^{x} \\
A_{s q}^{y}
\end{array}\right) \sin \left(k_{z, s q} z\right)+\left(\begin{array}{c}
B_{s q}^{x} \\
B_{s q}^{y}
\end{array}\right) \cos \left(k_{z, s q} z\right) \\
& \tilde{\mathbf{h}}_{t, s q}^{G a p}(z)=\left(\begin{array}{c}
D_{s q}^{x} \\
D_{s q}^{y}
\end{array}\right) \sin \left(k_{z, s q} z\right)+\left(\begin{array}{c}
F_{s q}^{x} \\
F_{s q}^{y}
\end{array}\right) \cos \left(k_{z, s q} z\right)
\end{aligned}
$$

where $k_{z, s q}=\left(k_{0}^{2}-k_{x, s}^{2}-k_{y, q}^{2}\right)^{1 / 2}$ is the vertical wavenumber of the $(s, q)^{t h}$ harmonic. This electromagnetic distribution is matched to the zero tangential E-field at the metallic parts of the PPW walls and to the tangential electromagnetic field distribution in the lateral waveguides

$$
\begin{array}{ll}
\mathbf{E}_{t}^{W G}\left(z=\frac{g}{2}\right) & =\sum_{m} r_{m}^{-} C_{m} \boldsymbol{\Phi}_{m}(x, y) \\
\mathbf{H}_{t}^{W G}\left(z=\frac{g}{2}\right) & =\sum_{m} r_{m}^{+} Y_{m} C_{m}\left[\hat{z} \times \boldsymbol{\Phi}_{m}(x, y)\right]
\end{array}
$$

where $C_{m}$ is the unknown coefficient of the $m^{t h}$ mode and $\boldsymbol{\Phi}_{m}$ and $Y_{m}$ are the corresponding cross-section modal function and the wave admittance. In the formulation both the $E$ - and $H$-field components should be matched at the lateral waveguide openings, by which it is possible to determine the unknown coefficients. One should note that the selected waveguide modes are orthogonal in PPW and in the lateral waveguide sections, but they are not mutually orthogonal. Therefore, the bi-Galerkin method is applied (i.e., waveguide modes of the PPW and of the lateral waveguides are used to test the $E$ - and $H$-field equations), and the mode-matching matrix is densely filled.

The generalized Bloch theorem [6], [26], [27] states that the field repeats itself (apart from an exponential factor) after a translation of half a period and a mirroring operation:

$$
\mathbf{E}(x, y,-z)= \pm e^{-j\left(k_{x, 0} \frac{p}{2}+k_{y, 0} \frac{p}{2}\right)} \mathbf{E}\left(x-\frac{p}{2}, y-\frac{p}{2}, z\right)
$$

If we assume that the field distribution in the lateral waveguides is described with only one waveguide mode, the linear system (whose determinant represents the characteristic equation of the mode travelling along the glide-symmetric periodic 
structure) is reduced to a single equation

$$
\begin{aligned}
& \sum_{\substack{s+q \\
\text { even }}} \tilde{\boldsymbol{\Phi}}\left(k_{x, s}, k_{y, q}\right) \tilde{\boldsymbol{\Phi}}\left(-k_{x, s},-k_{y, q}\right) \frac{k_{0}^{2}-k_{y, q}^{2}}{k_{z, s q}} \cot \left(\frac{k_{z, s q} g}{2}\right) \\
& -\sum_{\substack{s+q \\
\text { odd }}} \tilde{\boldsymbol{\Phi}}\left(k_{x, s}, k_{y, q}\right) \tilde{\boldsymbol{\Phi}}\left(-k_{x, s},-k_{y, q}\right) \frac{k_{0}^{2}-k_{y, q}^{2}}{k_{z, s q}} \tan \left(\frac{k_{z, s q} g}{2}\right)+j p^{2} \frac{r^{+}}{r^{-}} k_{z, 01}^{W G}=0
\end{aligned}
$$

The presence of the glide symmetry causes that, for even Floquet mode indices (i.e., for $s+q$ even number), the $E$-field in the parallel-plate region is described with $\cos \left(k_{z, s q} z\right)$ terms (i.e., with terms having even symmetry across the $z=0$ plane), while for odd Floquet mode indices, the $E$-field is described with $\sin \left(k_{z, s q} z\right)$ terms (i.e., with terms having odd symmetry; see eq. (4)).

It is interesting to compare this characteristic equation with the one given for an open holey surface. In the case of an open surface there is no mixing of odd and even modes, since there is no second holey surface forming the PPW (i.e., only the outgoing waves are present), so usually it is enough to consider only one PPW mode. The situation is different when a top ground plane approaches the structure [34], [35] or the structure possesses glide symmetry [26], [27].

\section{A. Dielectric glide-symmetric structures}

Until recently, most of the realized prototypes possessing higher symmetry were made from metal. However, in many applications and especially when going higher in frequency towards optics, dielectrics are the preferred building material. Although at first glance there are a lot of similarities between the analysis of metallic and dielectric glide-symmetric structures, the main difference comes from the fact that in the dielectric case part of the propagating wave (and thus part of the electromagnetic power) travels outside the dielectric waveguide. The wave propagating in the corrugated region can be modeled as a wave propagating along a periodic array of dielectric slabs, and it has to be matched at both interfaces with the modes present in PPW and in free-space, respectively [36]. For propagation constants smaller than the one of free-space, a fast wave is excited, which leads to leakage of electromagnetic energy (i.e., the considered structure actually represents a leaky-wave antenna).

\section{Glide SYMMETRY TO CREATE STOPBANDS AND EBGS (ELECTROMAGNETIC BANDGAPS)}

Some of the recently proposed structures with higher symmetries, and specifically those with glide symmetry, have been studied for their ability to produce stopbands or bandgaps. Periodic structures are often used in antenna designs to eliminate surface waves, to reduce the mutual coupling between antennas, or to produce microwave filters.

On the other hand, a new technology known as gap waveguide was recently proposed for high-frequency microwave circuits and antennas [13]. This technology requires stopbands for parallel-plate modes instead of surface waves (i.e., the periodic structure is embedded in a parallel-plate structure). One of the most popular types of gap waveguides is the groove gap waveguide technology. This technology is equivalent to a conventional rectangular waveguide in which the solid lateral walls are replaced by an EBG structure. Using this EBG structure, the waveguide is manufactured in two pieces that are assembled afterward by simply screwing them together. Electrical contact between the two pieces is not strictly required. 


\section{A. Analysis of two-dimensional holey glide-symmetric EBGs}

One possible solution to creating stopbands between parallel plates is to use periodic holes, as proposed in [37]. However, the stopband in all directions (i.e., EBG) created by holey structures typically is narrow, as is illustrated in Fig. 4 (a). Even after a thorough optimization of the constituent parameters of the holes, the stopband is quite different from one produced with pin-type structures [38].

In [39], it was demonstrated that holey structures with glide symmetry have a larger EBG bandwidth than conventional periodic holey structures. Indeed, the replacement of the top metal lid for a periodic structure made of holes, as shown in Fig. 4 (a), modifies all of the modes propagating in the structure and creates a wide stopband in between the second and third mode that can be used, for example, for gap waveguide technology [40], [41], [42].
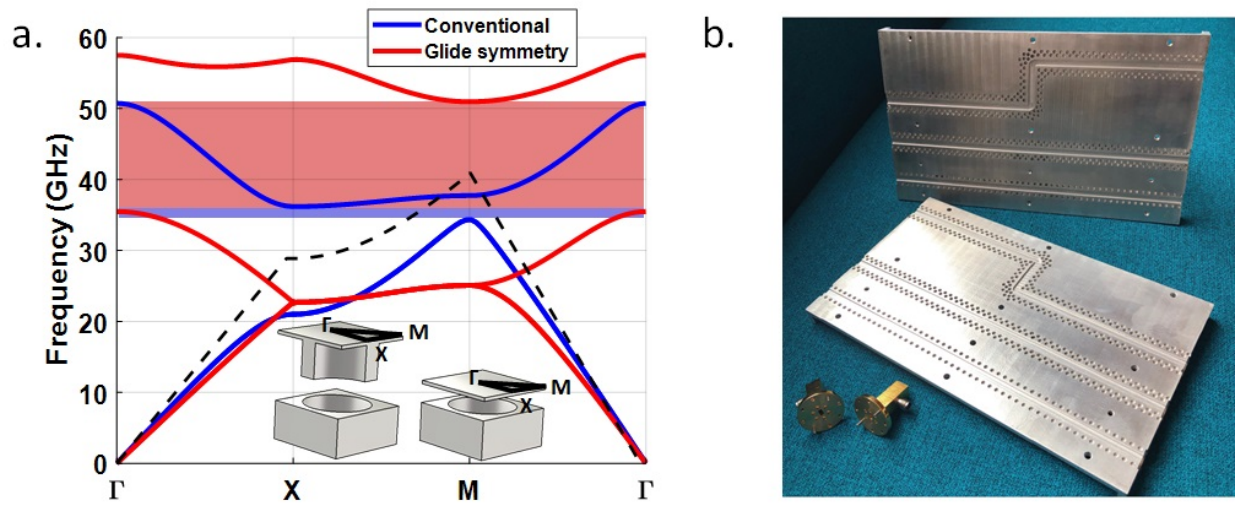

Fig. 4. (a) Comparison of the dispersion diagram for holey structures with and without glide symmetry. (b) Photo of a manufactured groove gap waveguide [40] and a flange [43] using glide-symmetric holes.

A complete parametric study of the glide-symmetric holey structures in terms of stopband properties was carried out in [38]. In this work, it was demonstrated that the period of the structure plays a key role in glide-symmetric holey structures since it determines the frequency range of operation. This implies that this periodic structure is electrically larger than those made of pins.

Another relevant conclusion is the fact that the depth of the holes affects the behavior of the structure up to a given height. This has interesting consequences for the manufacturing of the structure, since the depth of the holes does not need to be precisely controlled. This property can be explained by the fact that only evanescent modes penetrate the holes [34], [35]. These modes vanish after a given depth, making this structure practically insensitive to the flatness and height of the hole.

\section{B. Holey glide-symmetric structures for gap waveguide technology}

One popular application of EBGs is in a parallel-plate scenario for its potential use in gap waveguide technology. A number of periodic structures, typically pin-type, corrugations, and mushroom-type, have been proposed for gap waveguide technology [44]. Gap waveguides can be also implemented with glide-symmetric holes acting as an EBG [40]. The two main advantages of this implementation are simplified manufacturing and robustness. The presence of the glide-symmetric holey structure stops the leakage produced by the irregularities on the two surfaces, which are not perfectly attached in practice. 
With this approach, as is illustrated in the photo in Fig. 4 (b), it is possible to design waveguide components. For example, phase shifters were presented in [41] and [45]. Another example of a component made with this version of the technology is a $T E_{10}$ to $T E_{20}$ mode converter, which was used in [46] to produce a transversally compact slot array. Finally, a recent work [42] explored the possibilities of breaking the symmetry of the holes to add filtering capabilities to gap waveguide technology. When glide symmetry is broken, the bandgap is divided into two with a propagating mode in between. Therefore, in the frequency range of this mode, the waveguide has leakage. This leakage can be dissipated by introducing a thin lossy material between the layers. Recent implementations of glide symmetry also make use of multi-layer structures to reduce the manufacturing cost at high-frequency [47]. In this work, glide symmetry is imposed between adjacent layers. In other words, each layer is a half-unit cell displaced with respect to its top and bottom layers to increase the bandwidth of rejection.

\section{Glide symmetry for flanges}

Another interesting use of glide symmetry as an EBG was proposed in [43]. In this case, holey glide-symmetric holes were introduced in a waveguide flange to avoid leakage between connections. This technique can be used to produce fast measurements at very high frequencies since no physical contact between flanges is required. This idea was previously proposed with pins instead of holes in [15]. Here, again, the solution with holes is remarkably more robust and simple to manufacture than the pin-type. The concept was experimentally validated in the U-band in [43]. The designed flanges in this work are illustrated in the photo in Fig. 4 (b).

\section{Controllable stopbands on planar technology}

Although glide symmetry became popular for its immediate application to gap waveguide technology, its opportunities extend beyond this specific technology. For example, glide symmetry has also been applied to transmission lines. In particular, it was proposed in co-planar waveguides (CPW) to independently control the stopbands of even and odd modes [22]. Similarly, glide symmetry was employed in planar bifilar technology to control the stopbands generated by creating or breaking this symmetry [48]. In [49], elliptical holes between two dielectric layers were proposed to produce stopbands by breaking the glide symmetry. This work demonstrated that the width of these stopbands and their attenuation depends on the level of symmetry that is broken.

In [33], it was demonstrated that, using glide-symmetric mushrooms, the bandwidth of the first stopband in a microstrip technology can be increased without any additional manufacturing cost. This increase in the bandwidth is due to the different coupling that exists between conventional and glide-symmetric periodic unit cells. Therefore, only when the unit cells are electrically closed can glide symmetry be advantageous.

Finally, in [50], glide-symmetric holes were used to directly produce fully metallic filters. These filters are proposed as a robust and low-cost alternative to conventional waffle-iron technology for high-frequency applications, such as at the $\mathrm{K}_{a}$-band and beyond.

\section{GLIDE SYMMETRIES TO REDUCE DISPERSION}

Another important feature of higher symmetries, and in particular of glide symmetry, is that they can be used to reduce the dispersion of the first propagating mode in a periodic structure. This phenomenon has been used to create broadband lenses 
and low-dispersive transmission lines that find application in leaky-wave antennas.

\section{A. One-dimensional glide-symmetric structures}

The first studies on the dispersion of glide-symmetric structures were done for one-dimensional periodic structures [6]. When the coupling between sub-unit cells is strong, the dispersion of the modes propagating in the new periodic structure is reduced. More recently, these results were corroborated for thin metallic corrugations [18], planar bifilar lines [48], and slotted lines [21], [22]. In all of these studies, and for all these different technologies, when glide symmetry was applied, the first and second modes were connected, removing the first stopband [19]. The elimination of the first stopband inherently reduces the dispersion of the first mode, which means that the bandwidth is increased. This feature can be used, for example, to control the radiation of leaky-wave antennas [51], [22], [52], [53], [54]. remarkable antenna was proposed in [52] in which glide symmetry was used to compensate the phase dispersion of a leaky-wave antenna, producing a low-cost broadband antenna for point-to-point communications at $60 \mathrm{GHz}$.

Although the majority of the examples of glide symmetry use two layers to implement the symmetry, a new type of flat glide symmetry was proposed in [49]. In this work, elliptical holes were introduced between two dielectric layers. These holes modify the propagation characteristics of the parallel-plate modes inside each layer, and glide symmetry reduces their dispersion.

\section{B. Two-dimensional glide-symmetric structures}

Like one-dimensional periodic structures, two-dimensional glide-symmetric structures are less dispersive than conventional ones. In [25], it has been demonstrated that fully metallic glide-symmetric structures reduce the dispersion of the first propagating mode in PPW. Additionally, the first mode is able to produce higher refractive indexes and it is more isotropic. Although the first implementations of two-dimensional glide-symmetric structures were based on holey structures [25], similar properties were also found for glide-symmetric pins [55], [56]. This technique was employed in [57] to produce a broadband Luneburg lens antenna in the $\mathrm{K}_{a}$-band in for $5 \mathrm{G}$ communications. A photo of this antenna is plotted in Fig. 5 (a), and its radiation patterns at $28 \mathrm{GHz}$ are illustrated in Fig. 5 (b). This antenna is broadband, highly efficient, and has low scan losses.

Both pins and holes can be implemented to produce anisotropic responses. This anisotropy does not necessarily affects the broadband response of the periodic structures. Glide-symmetric anisotropic unit cells were used to compress the size of a conventional Luneburg lens with transformation optics [58], which is an asset in practical applications, since lens antennas can be reduced in size. In [58], it was demonstrated that glide symmetry was able to produce a higher level of compression than conventional unit cells.

Another example of two-dimensional glide symmetry can be found in [59]. In this work, the authors demonstrated that nearby layers of patches that possess glide symmetry are able to produce higher equivalent refractive indexes. Similarly, glide symmetry is a good candidate for producing dense materials that can be used for lens antennas [60], [61]. Other recent implementations of two-dimensional glide-symmetric structures include multi-layer glide-symmetric metasurfaces [62], [63], dielectric lenses [64], reconfigurable planar lenses in the optical regime [65], and broadband slow acoustic waves [66]. 
a.

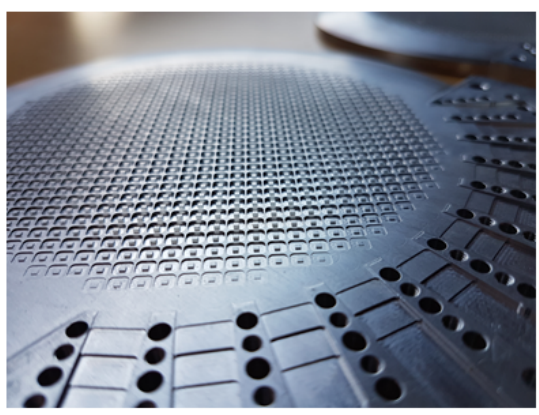

b.

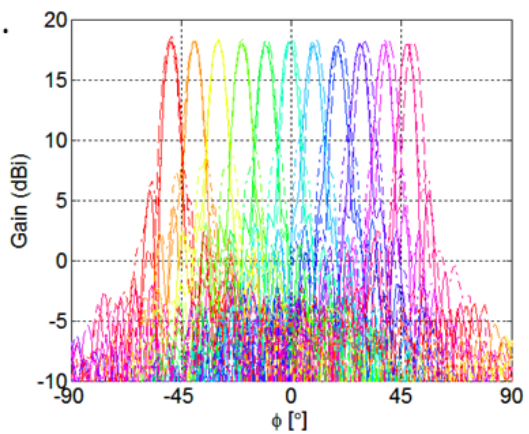

Fig. 5. Glide-symmetric Luneburg lens antenna [57]: a) Photo of the manufactured prototype. b) Simulated and measured radiation patterns at 28 GHz.

Finally, glide symmetry has been introduced to reduce the reflections at the contour of homogeneous lenses [67]. For example, in [67], it was demonstrated that glide-symmetric unit cells are able to produce effective magnetic materials to practically eliminate the reflections in a hyperbolic lens. This technique can be used to increase the total efficiency of lens antennas.

\section{TWIST-SYMMETRIC STRUCTURES}

Like glide symmetries, twist-symmetric structures have propagation properties which differ from common periodic structures [68]. They can also be described with analogous models to glide symmetry. In [6], it was demonstrated that an $m$-fold twist line leads to the suppression of the first $m-1$ stopbands in its Brillouin diagram. As in glide-symmmetric structures, this effect can be used to reduce frequency dispersion.

Following the generalized Floquet theorem, $m$-fold twist-symmetric structures can be characterized by a sub-unit cell of length $p / m$. Therefore, they can be analyzed with equivalent-circuit models as in Section IV-A [24] and with a multi-modal approach similar to the one described in Section IV-B [31]. The latter method requires the description of a sub-unit cell as a $2 N$-port network, where $N$ is the number of background modes retained at each Floquet boundary, which defines a sub-cell transmission matrix $\boldsymbol{T}_{1 / m}$ as in (2). Unlike with glide symmetry, in twist structures, the modes are classed according to their azimuthal variation on the circular cross-section of the waveguide $\left(\cos (n \varphi)\right.$ and $\sin (n \varphi)$, for a $n^{\text {th }}$ azimuthal order. The modes are then rotated an angle $\pi / m$ by means of a rotation matrix every translation of $p / m$. The eigenvalue problem in (1) can be formulated as [31]:

$$
\left[\begin{array}{ll}
\boldsymbol{Q} & \mathbf{0} \\
\mathbf{0} & \boldsymbol{Q}
\end{array}\right] \cdot \boldsymbol{T}_{1 / m} \cdot\left[\begin{array}{l}
\boldsymbol{V} \\
\boldsymbol{I}
\end{array}\right]=e^{-j k_{z} \frac{p}{m}}\left[\begin{array}{l}
\boldsymbol{V} \\
\boldsymbol{I}
\end{array}\right]
$$

where the $Q$ matrix rotates the background mode at the sub-unit cell ports. Here, again, the presence of higher-order modes leads to a different dispersive behavior with respect to a line without a twist operation, where the $Q$ matrix would become an identity matrix in (8). 


\section{A. Twist symmetries in circular-section coaxial cables}

Twist symmetry has been used in coaxial lines to increase their density over a large bandwidth of operation [17], [23]. Two alternatives have been studied: holey structures [23] and pins [17], [24]. Examples of the latter are illustrated in Fig. 6 (a). As illustrated in Fig. 6 (b), the effective density of the medium depends on the order of the twist symmetry. Holes drilled in the inner conductor of the coaxial cable also lead to a similar effect, as demonstrated numerically and experimentally in [23]. In [23], to increase the effect of the holes in the line, an opening of $\pi$ radians was chosen. In [24], $\pi$-radian rings were added to the outer conductor in a twist configuration, and an extension of the non-dispersive range of frequency was clearly observed when the twist order increased. As in glide structures, the physical parameters of the scatterers along the line can be changed, thus obtaining a graded-index line where different frequencies can be rejected at different positions of the transmission line [23].

In circular coaxial cables, another kind of symmetry was introduced which was analogous with the glide operator: polar glide symmetry. This symmetry is created by translating by half a period the obstacle in one conductor and mirroring it into the other conductor [17], [23], [24]. This operation is not rigorously a glide symmetry, and it does not close the first stopband at the edge of the Brillouin zone. However, a fine tuning of the structure can lead in waveguides with circular cross-sections to similar effects as glide symmetry in Cartesian coordinates. Polar glide with $\pi$-radian rings was employed in [23] to prove this effect. In [24], a polar glide was implemented with $\pi$-radian rings, which are equivalent to a stepped discontinuity in the conductor radius. In its polar glide configuration, this stepped discontinuity alternates between the inner and the outer conductor radii.

a.

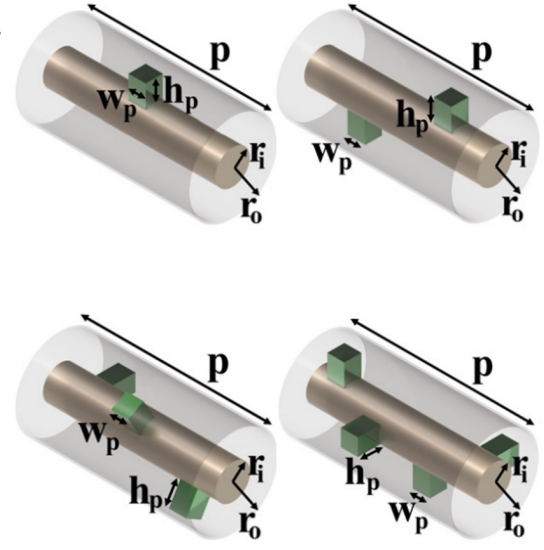

b.

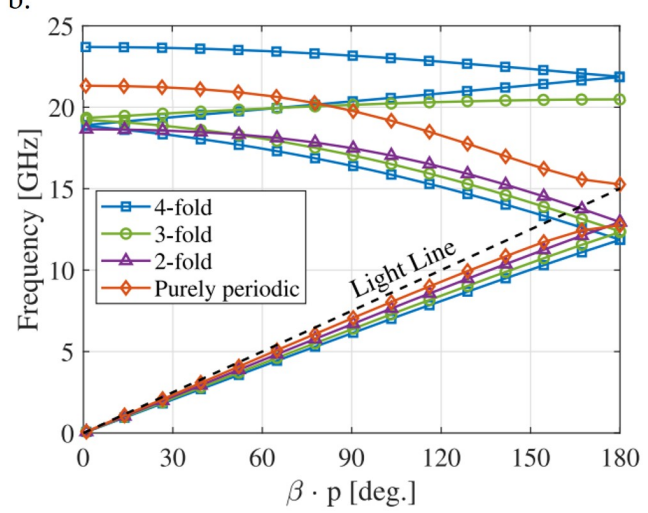

Fig. 6. a) Coaxial cable loaded with a periodic array of pins with 1-, 2-, 3-, and 4-fold twist symmetry. b) Dispersion diagrams of these coaxial cables [68].

Another original way to combine twist and glide symmetries was proposed in [69], where an helicoidal radiating line (i.e., an helix antenna) is perturbed with periodic corrugations. A sequence of periodic corrugations naturally defines a twist symmetry due to the helicoidal shape of the line. Furthermore, a glide-symmetric sequence of corrugations can also be combined with the twist symmetry of the line. As a result, several geometric parameters can be used to tune the propagation features along the 
line with a broadband response. In turn, in [69], it was proved that an helix antenna can be miniaturized without a significant deterioration of its performance.

\section{B. Twist-symmetric waveguides and metasurfaces}

Twist symmetries have also been implemented in circular waveguides [70], as the ones illustrated in Fig. 7. In this work, a holey waveguide was periodically loaded with metallic sheets with perforated holes of circular shape that are not centered in the waveguide cross-section. These obstacles block the first TE mode of the waveguide and let only TM modes propagate. Furthermore, if these circular holes are rotated in a twist-symmetric feature, the stopbands between the first $n$ modes are suppressed. Acting on geometrical parameters, a broader band of propagation is available as the period decreases, thus leading to a miniaturization of the line. Finally, if the holes have an elliptical shape, only a twist-symmetric rotation will open a passband, as illustrated in Fig. 7.

a.

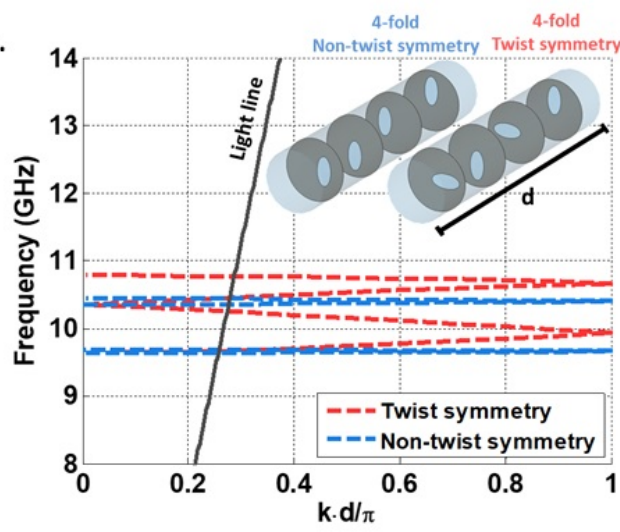

b.

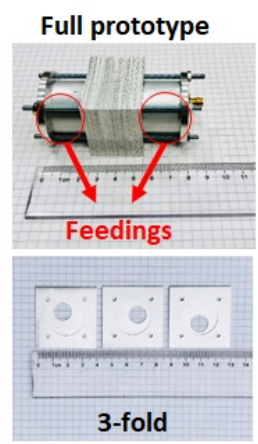

Fig. 7. Circular waveguide with twist-symmetric irises as in [70]. a) Dispersion diagrams for a 4-fold twist-symmetric configuration with elliptical holes. b) Photos of the prototype for circular holes.

Other applications of twist symmetry can be encountered in metasurface designs. For example, in [71], a multilayered configuration of metasurfaces based on split-ring resonators was proposed. The layered structure is locally twist-symmetric with respect to the stratification direction. The electric density seen by a plane wave traveling across the metasurfaces depends on the twist order. These properties can be used to tailor a flat lens, since different phase delays at each incidence point can be produced. In [71], these delays were designed to obtain a plane wave emerging from the lens. The design maintained the orientation of the split rings on the first and the last metasurfaces, so no depolarization is encountered. However, similar configurations can be used to convert the polarization [72], [73].

\section{CONCLUSION}

In this paper, we have described the latest discoveries on higher symmetries and their opportunities for designing electromagnetic devices. There are two known types of spatial higher symmetries: glide and twist. Glide symmetry has been the most broadly studied since it can be implemented in planar structures, which are easy to manufacture. 
For example, glide symmetry has been proposed to reduce the dispersion properties of the first propagating mode in parallelplate configurations. This opened possibilities for increasing the bandwidth of metasurface lens antennas. More recently, glide symmetry has been proposed to reduce the cost of low-dispersive leaky-wave antennas.

Additionally, glide symmetry has been reported to increase the bandwidth of operation of conventional EBGs. This opens opportunities, for example, for cost-effective and robust gap waveguide technology and filters, and to avoid undesireable leakage in flanges. Glide symmetry has been also proposed to produce tunable stopbands and to increase the bandwidth of filters.

In more recent works, glide symmetry has been found suitable for increasing the anisotropy of periodic structures, which can be used to reduce the dimensions of lens antennas. Finally, glide symmetry has been proposed for reducing the reflections at the contour of hyperbolic lenses, which can be used to increase the total efficiency of lens antennas.

Twist symmetries have been less studied than glide symmetries due to the complexity of their practical implementation. However, like glide symmetries, they have been proposed to reduce the dispersion of propagating modes, and to control stopbands in transmission lines, waveguides, and flat metasurfaces.

\section{ACKNOWLEDGMENT}

The authors would like to thank O. Zetterström for providing the photo represented in Fig. 5 (a).

\section{REFERENCES}

[1] A. Okabe, B. Boots, K. Sugihara, S. N. Chiu, and D. G. Kendall, Spatial Tessellations: Concepts and Applications of Voronoi Diagrams. John Wiley Sons, Ltd, 1998.

[2] G. L. Trigg, "Higher symmetries," Physical Review Letters, vol. 14, pp. 479-479, Mar 1965.

[3] P. J. Crepeau and P. R. McIsaac, "Consequences of symmetry in periodic structures," Proceedings of the IEEE, vol. 52, no. 1, pp. 33-43, Jan 1964.

[4] R. Mittra and S. Laxpati, "Propagation in a wave guide with glide reflection symmetry," Canadian Journal of Physics, vol. 43, no. 2, pp. 353-372, 1965.

[5] R. Kieburtz and J. Impagliazzo, "Multimode propagation on radiating traveling-wave structures with glide-symmetric excitation," IEEE Transactions on Antennas and Propagation, vol. 18, no. 1, pp. 3-7, Jan 1970.

[6] A. Hessel, M. H. Chen, R. C. M. Li, and A. A. Oliner, "Propagation in periodically loaded waveguides with higher symmetries," Proceedings of the IEEE, vol. 61, no. 2, pp. 183-195, Feb 1973.

[7] A. Sihvola, Electromagnetic Emergence in Metamaterials. Dordrecht: Springer Netherlands, 2002, pp. 3-17.

[8] N. Engheta and R.-W. Ziolkowski, Metamaterials: Physics and Engineering Explorations. Institute of Electrical and Electronics Engineers, Inc., 2006.

[9] S. Maci, G. Minatti, M. Casaletti, and M. Bosiljevac, "Metasurfing: Addressing waves on impenetrable metasurfaces," IEEE Antennas and Wireless Propagation Letters, vol. 10, pp. 1499-1502, 2011.

[10] C. Pfeiffer and A. Grbic, "Metamaterial huygens' surfaces: Tailoring wave fronts with reflectionless sheets," Phys. Rev. Lett., vol. 110, p. 197401, May 2013.

[11] The Evolution of Electromagnetic Waveguides: From Hollow Metallic Guides to Microwave Integrated Circuits. John Wiley Sons, Ltd, 2006, ch. 16, pp. 543-566.

[12] Y. Wang, J. Li, L. Huang, Y. Jing, A. Georgakopoulos, and P. Demestichas, "5G mobile: Spectrum broadening to higher-frequency bands to support high data rates," IEEE Vehicular Technology Magazine, vol. 9, no. 3, pp. 39-46, Sep. 2014.

[13] P.-S. Kildal, E. Alfonso, A. Valero-Nogueira, and E. Rajo-Iglesias, "Local metamaterial-based waveguides in gaps between parallel metal plates," IEEE Antennas and Wireless Propagation Letters, vol. 8, pp. 84-87, 2009.

[14] O. Quevedo-Teruel, M. Ebrahimpouri, and F. Ghasemifard, "Lens antennas for 5G communications systems," IEEE Communications Magazine, vol. 56, no. 7, pp. 36-41, July 2018.

[15] S. Rahiminejad, E. Pucci, V. Vassilev, P.-S. Kildal, S. Haasl, and P. Enoksson, "Polymer gap adapter for contactless, robust, and fast measurements at 220-325 GHz," Journal of Microelectromechanical Systems, vol. 25, no. 1, pp. 160-169, Feb 2016. 
[16] S. Amari, R. Vahldieck, and J. Bornemann, "Accurate analysis of periodic structures with an additional symmetry in the unit cell from classical matrix eigenvalues," IEEE Transactions on Microwave Theory and Techniques, vol. 46, no. 10, pp. 1513-1515, Oct 1998.

[17] O. Dahlberg, R. C. Mitchell-Thomas, and O. Quevedo-Teruel, "Reducing the dispersion of periodic structures with twist and polar glide symmetries," Scientific Reports, vol. 7, no. 1, p. 10136, 82017.

[18] R. Quesada, D. Martín-Cano, F. J. García-Vidal, and J. Bravo-Abad, "Deep-subwavelength negative-index waveguiding enabled by coupled conformal surface plasmons," Optics Letters, vol. 39, no. 10, pp. 2990-2993, May 2014.

[19] G. Valerio, Z. Sipus, A. Grbic, and O. Quevedo-Teruel, "Accurate equivalent-circuit descriptions of thin glide-symmetric corrugated metasurfaces," IEEE Transactions on Antennas and Propagation, vol. 65, no. 5, pp. 2695-2700, May 2017.

[20] F. Ghasemifard, M. Norgren, and O. Quevedo-Teruel, "Dispersion analysis of 2-D glide-symmetric corrugated metasurfaces using mode-matching technique," IEEE Microwave and Wireless Components Letters, vol. 28, no. 1, pp. 1-3, Jan 2018.

[21] M. Camacho, R. C. Mitchell-Thomas, A. P. Hibbins, J. R. Sambles, and O. Quevedo-Teruel, "Designer surface plasmon dispersion on a one-dimensional periodic slot metasurface with glide symmetry," Optics Letters, vol. 42, no. 17, pp. 3375-3378, Sep 2017.

[22] — "Mimicking glide symmetry dispersion with coupled slot metasurfaces," Applied Physics Letters, vol. 111, no. 12 , p. 121603 , 2017.

[23] F. Ghasemifard, M. Norgren, and O. Quevedo-Teruel, "Twist and polar glide symmetries: an additional degree of freedom to control the propagation characteristics of periodic structures," Scientific Reports, vol. 8, no. 11266, July 2018.

[24] Q. Chen, F. Ghasemifard, G. Valerio, and O. Quevedo-Teruel, "Modeling and dispersion analysis of coaxial lines with higher symmetries," IEEE Transactions on Microwave Theory and Techniques, vol. 66, no. 10, pp. 4338-4345, Oct 2018.

[25] O. Quevedo-Teruel, M. Ebrahimpouri, and M. N. M. Kehn, "Ultrawideband metasurface lenses based on off-shifted opposite layers," IEEE Antennas and Wireless Propagation Letters, vol. 15, pp. 484-487, Dec 2016.

[26] G. Valerio, F. Ghasemifard, Z. Sipus, and O. Quevedo-Teruel, "Glide-symmetric all-metal holey metasurfaces for low-dispersive artificial materials: Modeling and properties," IEEE Transactions on Microwave Theory and Techniques, vol. 66, no. 7, pp. 3210-3223, July 2018.

[27] F. Ghasemifard, M. Norgren, O. Quevedo-Teruel, and G. Valerio, “Analyzing glide-symmetric holey metasurfaces using a generalized Floquet theorem,” IEEE Access, vol. 6, pp. 71 743-71750, 2018

[28] S. Sensiper, "Electromagnetic wave propagation on helical structures (a review and survey of recent progress)," Proceedings of the IRE, vol. 43, no. 2, pp. 149-161, Feb 1955.

[29] M. Bagheriasl, O. Quevedo-Teruel, and G. Valerio, "Bloch analysis of artificial lines and surfaces exhibiting glide symmetry," IEEE Transactions on Microwave Theory and Techniques, vol. 67, no. 7, July 2019.

[30] F. Mesa, R. Rodríguez-Berral, and F. Medina, "On the computation of the dispersion diagram of symmetric one-dimensionally periodic structures," Symmetry, vol. 10, no. 8, 2018.

[31] M. Bagheriasl and G. Valerio, "Bloch analysis of electromagnetic waves in twist-symmetric lines," Symmetry, vol. 5, no. 1, p. 620, 2019.

[32] N. Marcuvitz, Waveguide Handbook. Isha Books, 2013.

[33] B. A. Mouris, A. Fernandez-Prieto, R. Thobaben, J. Martel, F. Mesa, and O. Quevedo-Teruel, "On the increment of the bandwidth of mushroom-type EBG structures with glide symmetry," IEEE Transactions on Microwave Theory Technology, in press.

[34] F. J. García de Abajo and J. J. Sáenz, "Electromagnetic surface modes in structured perfect-conductor surfaces," Physical Review Letters, vol. 95, p. 233901, Nov 2005

[35] G. Valerio, Z. Sipus, A. Grbic, and O. Quevedo-Teruel, "Nonresonant modes in plasmonic holey metasurfaces for the design of artificial flat lenses," Optics Letters, vol. 42, no. 10, pp. 2026-2029, May 2017.

[36] Z. Sipus and M. Bosiljevac, "Modelling of glide-symmetric dielectric structures," Symmetry, vol. 11, 2019.

[37] D. Dawn, Y. Ohashi, and T. Shimura, "A novel electromagnetic bandgap metal plate for parallel plate mode suppression in shielded structures," IEEE Microwave and Wireless Components Letters, vol. 12, no. 5, pp. 166-168, May 2002.

[38] M. Ebrahimpouri, O. Quevedo-Teruel, and E. Rajo-Iglesias, "Design guidelines for gap waveguide technology based on glide-symmetric holey structures," IEEE Microwave and Wireless Components Letters, vol. 27, no. 6, pp. 542-544, June 2017.

[39] M. Ebrahimpouri, E. Rajo-Iglesias, Z. Sipus, and O. Quevedo-Teruel, "Low-cost metasurface using glide symmetry for integrated waveguides," in 2016 10th European Conference on Antennas and Propagation (EuCAP), April 2016, pp. 1-2.

[40] - "Cost-effective gap waveguide technology based on glide-symmetric holey EBG structures," IEEE Transactions on Microwave Theory and Techniques, vol. 66, no. 2, pp. 927-934, Feb 2018. 
[41] E. Rajo-Iglesias, M. Ebrahimpouri, and O. Quevedo-Teruel, "Wideband phase shifter in groove gap waveguide technology implemented with glidesymmetric holey EBG," IEEE Microwave and Wireless Components Letters, vol. 28, no. 6, pp. 476-478, June 2018.

[42] P. Padilla, A. Palomares-Caballero, A. Alex-Amor, J. Valenzuela-Valdés, J. M. Fernández-González, and O. Quevedo-Teruel, "Broken glide-symmetric holey structures for bandgap selection in gap-waveguide technology," IEEE Microwave and Wireless Components Letters, vol. 29, no. 5, pp. 327-329, May 2019.

[43] M. Ebrahimpouri, A. A. Brazalez, L. Manholm, and O. Quevedo-Teruel, "Using glide-symmetric holes to reduce leakage between waveguide flanges," IEEE Microwave and Wireless Components Letters, vol. 28, no. 6, pp. 473-475, June 2018.

[44] E. Rajo-Iglesias and P.-S. Kildal, "Numerical studies of bandwidth of parallel-plate cut-off realised by a bed of nails, corrugations and mushroom-type electromagnetic bandgap for use in gap waveguides," IET Microwaves, Antennas Propagation, vol. 5, no. 3, pp. 282-289, Feb 2011.

[45] A. Palomares-Caballero, A. Alex-Amor, P. Padilla, F. Luna, and J. Valenzuela-Valdes, "Compact and low-loss V-band waveguide phase shifter based on glide-symmetric pin configuration,” IEEE Access, vol. 7, pp. 31 297-31 304, 2019.

[46] Q. Liao, E. Rajo-Iglesias, and O. Quevedo-Teruel, "Ka-band fully metallic TE 40 slot array antenna with glide-symmetric gap waveguide technology," IEEE Transactions on Antennas and Propagation, vol. 67, no. 10, pp. 6410-6418, Oct 2019.

[47] A. Vosoogh, H. Zirath, and Z. S. He, "Novel air-filled waveguide transmission line based on multilayer thin metal plates," IEEE Transactions on Terahertz Science and Technology, vol. 9, no. 3, pp. 282-290, May 2019.

[48] P. Padilla, L. F. Herrán, A. Tamayo-Domínguez, J. F. Valenzuela-Valdés, and O. Quevedo-Teruel, "Glide symmetry to prevent the lowest stopband of printed corrugated transmission lines," IEEE Microwave and Wireless Components Letters, vol. 28, no. 9, pp. 750-752, Sep. 2018.

[49] A. Tamayo-Dominguez, J.-M. Fernandez-Gonzalez, and O. Quevedo-Teruel, "One-plane glide-symmetric holey structures for stop-band and refraction index reconfiguration," Symmetry, vol. 11, no. 4, 2019.

[50] A. Monje-Real, N. J. G. Fonseca, O. Zetterstrom, E. Pucci, and O. Quevedo-Teruel, "Holey glide-symmetric filters for 5G at millimeter-wave frequencies," IEEE Microwave and Wireless Components Letters, vol. 30, no. 1, pp. 31-34, Jan 2020.

[51] J. J. Wu, C. Wu, D. J. Hou, K. Liu, and T. Yang, "Propagation of low-frequency spoof surface plasmon polaritons in a bilateral cross-metal diaphragm channel waveguide in the absence of bandgap," IEEE Photonics Journal, vol. 7, no. 1, pp. 1-8, Feb 2015.

[52] Q. Chen, O. Zetterstrom, E. Pucci, A. Palomares-Caballero, P. Padilla, and O. Quevedo-Teruel, "Glide-symmetric holey leaky-wave antenna with low dispersion for 60-GHz point-to-point communications," IEEE Transactions on Antennas and Propagation, in press.

[53] J. Chen, W. Yuan, C. Zhang, W. X. Tang, L. Wang, Q. Cheng, and T. J. Cui, "Wideband leaky-wave antennas loaded with gradient metasurface for fixed-beam radiations with customized tilting angles," IEEE Transactions on Antennas and Propagation, in press.

[54] G. Zhang, Q. Zhang, Y. Chen, and R. D. Murch, "High-scanning-rate and wide-angle leaky-wave antennas based on glide-symmetry goubau line," IEEE Transactions on Antennas and Propagation, in press.

[55] N. Memeletzoglou, C. Sanchez-Cabello, F. Pizarro-Torres, and E. Rajo-Iglesias, "Analysis of periodic structures made of pins inside a parallel plate waveguide," Symmetry, vol. 11, no. 4, 2019.

[56] D. Sun, X. Chen, J. Deng, L. Guo, W. Cui, K. Yin, Z. Chen, C. Yao, and F. Huang, "Gap waveguide with interdigital-pin bed of nails for high-frequency applications," IEEE Transactions on Microwave Theory and Techniques, vol. 67, no. 7, pp. 2640-2648, July 2019.

[57] O. Quevedo-Teruel, J. Miao, M. Mattsson, A. Algaba-Brazalez, M. Johansson, and L. Manholm, "Glide-symmetric fully metallic Luneburg lens for 5G communications at Ka-band," IEEE Antennas and Wireless Propagation Letters, vol. 17, no. 9, pp. 1588-1592, Sept 2018.

[58] M. Ebrahimpouri and O. Quevedo-Teruel, "Ultrawideband anisotropic glide-symmetric metasurfaces," IEEE Antennas and Wireless Propagation Letters, vol. 18, no. 8, pp. 1547-1551, Aug 2019.

[59] T. Chang, J. U. Kim, S. K. Kang, H. Kim, D. K. Kim, Y.-H. Lee, and J. Shin, "Broadband giant-refractive-index material based on mesoscopic space-filling curves," Nature Communications, vol. 7, Article number 12661, 2016.

[60] D. Cavallo and C. Felita, "Analytical formulas for artificial dielectrics with nonaligned layers," IEEE Transactions on Antennas and Propagation, vol. 65, no. 10, pp. 5303-5311, Oct 2017.

[61] D. Cavallo, "Dissipation losses in artificial dielectric layers," IEEE Transactions on Antennas and Propagation, vol. 66, no. 12, pp. 7460-7465, Dec 2018.

[62] J. D. de Pineda, R. C. Mitchell-Thomas, A. P. Hibbins, and J. R. Sambles, "A broadband metasurface Luneburg lens for microwave surface waves," Applied Physics Letters, vol. 111, no. 21, p. 211603, 2017. 
[63] J. D. de Pineda, A. P. Hibbins, and J. R. Sambles, "Microwave edge modes on a metasurface with glide symmetry," Physical Review B, vol. 98, p. 205426, Nov 2018.

[64] D. Jia, Y. He, N. Ding, J. Zhou, B. Du, and W. Zhang, "Beam-steering flat lens antenna based on multilayer gradient index metamaterials," IEEE Antennas and Wireless Propagation Letters, vol. 17, no. 8, pp. 1510-1514, Aug 2018.

[65] M. M. Shanei, D. Fathi, F. Ghasemifard, and O. Quevedo-Teruel, "All-silicon reconfigurable metasurfaces for multifunction and tunable performance at optical frequencies based on glide symmetry," Scientific Reports, vol. 9, no. 1, pp. 2045-2322, Sept 2019.

[66] J. G. Beadle, I. R. Hooper, J. R. Sambles, and A. P. Hibbins, "Broadband, slow sound on a glide-symmetric meander-channel surface," The Journal of the Acoustical Society of America, vol. 145, no. 5, pp. 3190-3194, 2019.

[67] M. Ebrahimpouri, L. F. Herran, and O. Quevedo-Teruel, "Wide-angle impedance matching using glide-symmetric metasurfaces," IEEE Microwave and Wireless Components Letters, vol. 30, no. 1, pp. 8-11, Jan 2020.

[68] O. Dahlberg, F. Ghasemifard, G. Valerio, and O. Quevedo-Teruel, "Propagation characteristics of periodic structures possessing twist and polar glide symmetries," EPJ Applied Metamaterials, vol. 6, p. 14, 2019.

[69] A. Palomares-Caballero, P. Padilla, A. Alex-Amor, J. Valenzuela-Valdés, and O. Quevedo-Teruel, "Twist and glide symmetries for helix antenna design and miniaturization," Symmetry, vol. 11, no. 3, 2019.

[70] O. Quevedo-Teruel, O. Dahlberg, and G. Valerio, "Propagation in waveguides with transversal twist-symmetric holey metallic plates," IEEE Microwave and Wireless Components Letters, vol. 28, no. 10, pp. 858-860, Oct 2018.

[71] O. Dahlberg, G. Valerio, and O. Quevedo-Teruel, "Fully metallic flat lens based on locally twist-symmetric array of complementary split-ring resonators," Symmetry, vol. 11, no. 4, 2019.

[72] Z. Wei, Y. Cao, Y. Fan, X. Yu, and H. Li, "Broadband polarization transformation via enhanced asymmetric transmission through arrays of twisted complementary split-ring resonators," Applied Physics Letters, vol. 99, no. 22, p. 221907, 2011.

[73] A. N. Askarpour, Y. Zhao, and A. Alù, "Wave propagation in twisted metamaterials," Physical Review B, vol. 90, p. 054305, Aug 2014. 